\title{
Simulation of cross contamination and decontamination of Campylobacter jejuni during handling of contaminated raw vegetables in a domestic kitchen
}

\begin{abstract}
Campylobacter jejuni was found to occur at high prevalence in the raw salad vegetables examined. Previous reports describe cross-contamination involving meat; here we investigated the occurrence of cross-contamination and decontamination events in the domestic kitchen via C. jejuni-contaminated vegetables during salad preparation. This is the first report concerning quantitative cross-contamination and decontamination involving naturally contaminated produce. The study was designed to simulate the real preparation of salad in a household kitchen, starting with washing the vegetables in tap water, then cutting the vegetables on a cutting board, followed by slicing cucumber and blanching (heating in hot water) the vegetables in $85^{\circ} \mathrm{C}$ water. Vegetables naturally contaminated with C. jejuni were used throughout the simulation to attain realistic quantitative data. The mean of the percent transfer rates for C. jejuni from vegetable to wash water was 30.1 to $38.2 \%$; from wash water to cucumber, it was 26.3 to $47.2 \%$; from vegetables to cutting board, it was 1.6 to $10.3 \%$; and from cutting board to cucumber, it was 22.6 to $73.3 \%$. The data suggest the wash water and plastic cutting board as potential risk factors in C. jejuni transmission to consumers. Washing of the vegetables with tap water caused a 0.4-log reduction of $C$. jejuni attached to the vegetables (most probable number/gram), while rapid blanching reduced the number of $\mathrm{C}$. jejuni organisms to an undetectable level.
\end{abstract}

